\title{
ANÁLISE DE AGRUPAMENTO DE 16 GÊNEROS E 71 ESPÉCIES DE MYRTEAE, COM BASE EM DADOS DA ANATOMIA DA MADEIRA ${ }^{1}$
}

\author{
SIDINEI RODRIGUES DOS SANTOS² ANELISE MARTA SIEGLOCH ${ }^{3}$ \\ JOSÉ NEWTON CARDOSO MARCHIORI ${ }^{4}$
}

\begin{abstract}
RESUMO
A Tribo Myrteae (antiga subfamília Myrtoideae) é uma das mais importantes e complexas de Myrtaceae, fato que pode ser comprovado pelo grande número de alterações na classificação interna, ocorrida ao longo do tempo. No presente estudo é realizada uma análise de agrupamento com base em dados anatômicos da madeira, visando ao reconhecimento de padrões úteis ao esclarecimento da correta circunscrição dos gêneros e grupos superiores da referida Tribo. O trabalho incluiu informações anatômicas de 16 gêneros e 71 espécies brasileiras de Myrteae, colhidas da literatura. Todas as espécies estudadas agruparam-se a menos de 0,5 de dissimilaridade, indicando uma grande homogeneidade estrutural. Observou-se uma distribuição difusa das espécies e gêneros no dendrograma, não compatível com qualquer classificação existente. A falta de delimitação para os gêneros e grupos superiores, com exceção de Myrceugenia, limita a opinião da Anatomia da Madeira em discussões taxonômicas em Myrteae.

Palavras-chave: Anatomia da madeira, Análise de agrupamento, Myrteae, Taxonomia.

\section{ABSTRACT}

[Cluster analysis of 16 genus and 71 species of Myrteae, based on wood anatomy data].

The Myrteae (formerly subfamily Myrtoideae) is one of the most important and complex tribes of Myrtaceae, a fact that can be proven by the large number of changes in its internal classification along time. This paper deals with a cluster analysis of Myrteae, with the aim to contribute to clarify the correct circumscription of genera and higher groups. The study included wood anatomical data from 16 genera and 71 species of Brazilian Myrteae, collected in the literature. All species were grouped up to 0.5 dissimilarity, indicating a large structural homogeneity. There was also a diffuse distribution of species and genera in the dendrogram, not compatible with any existing classification. The lack of definition for the genera and higher groups, except to Myrceugenia, limits the opinion of Wood Anatomy in taxonomic discussions on Myrteae.
\end{abstract}

Key words: Cluster analysis, Myrteae, Taxonomy, Wood anatomy.

\section{INTRODUÇÃO}

Com cerca de 150 gêneros e 3600 espécies de árvores e arbustos, a família Myrtaceae é uma das mais importantes da flora lenhosa da América do Sul, onde se encontra o seu principal centro de dispersão (Govaerts et al., 2008). Bem representado, o Brasil conta com aproximadamente 23 gêneros e 1012 espécies nativas, to-

1 Recebido para publicação em 12/10/2014 e aceito para publicação em 21/02/2015.

2 Biólogo. Doutor. Universidade Federal do Pampa. Dom Pedrito, RS, Brasil.sthurt.bio@gmail.com

3 Engenheira Florestal, Doutoranda do PPGEF - UFSM (Bolsista CAPES), Santa Maria, RS, Brasil.

4 Engenheiro Florestal, Doutor. Professor Titular, UFSM, Santa Maria, RS, Brasil. das da tribo Myrteae ou subfamília Myrtoideae, de acordo com classificação mais antiga (Sobral et al., 2014).

Apesar da relativa facilidade com que a família é reconhecida por especialistas, a identificação e delimitação das espécies e gêneros se mostra, por vezes, bastante difícil (Landrum e Kawasaki, 1997). Como resultado, a classificação interna do grupo tem sofrido importantes alterações nos últimos anos (Sobral, 2003).

A circunscrição dos grandes grupos infrafamiliares em Myrtaceae tornou-se melhor compreendida nos últimos anos, principalmente a partir do desenvolvimento de estudos filogenéticos baseados em análise de DNA (Johnson e Briggs, 1984; Wilson et al., 2001; 
2005; Lucas et al., 2005; 2007; Costa, 2009). Tais estudos demonstram que as tradicionais subfamílias Myrtoideae e Leptospermoideae, bem como as subtribos propostas por Berg (1855-56; 1857-59), não formam grupos naturais, motivo pelo qual merecem ser descartadas. $\mathrm{O}$ mesmo se pode dizer a respeito da validade e/ou abrangência de alguns gêneros, tais como Hexachlamys e Eugenia, Neomitranthes e Siphoneugena, Plinia e Myrciaria, Myrcia, Gomidesia, Calyptranthes e Marlierea, entre outros, que também passaram por revisões, embora ainda sem consenso (Landrum e Kawasaki, 1997; Sobral, 2003; Lucas e Sobral, 2011; Lucas et al., 2011).

Atualmente, são reconhecidas duas subfamílias (Myrtoideae e Psiloxyloideae) e 17 tribos, incluindo Myrteae, à qual pertence todos os taxa de frutos carnosos nativos na América do Sul (Wilson et al., 2005). A tribo Myrteae, por sua vez, conta com pelo menos seis grupamentos genéricos na concepção mais recente (Lucas et al., 2005; 2007; Costa, 2009).

Vários estudos, em diversas áreas, têm sido realizados para auxiliar no esclarecimento destas questões, incluindo anatomia da madeira. Détienne e Jacquet (1983) investigaram a estrutura anatômica do lenho de 8 gêneros e 14 espécies de Myrtaceae da Amazônia, com vistas ao reconhecimento de caracteres úteis à identificação. Os autores concluíram que é difícil a separação dos gêneros, seja pela homogeneidade dos mesmos, seja pela heterogeneidade entre espécies de um mesmo gênero. Dias-Leme et al. (1995), em estudo anatômico envolvendo 31 espécies, de 4 gêneros da subtribo Myrciinae, não encontraram, igualmente, nenhuma característica ou conjunto de características de valor diagnóstico a este nível taxonômico, a exemplo de Santos e Marchiori (2011a) e Santos et al. (2013).

Apesar dos esforços, que têm resultado em avanços consideráveis sobre a taxonomia das Myrtaceae, ainda resta muito a ser investigado, até que se possa estabelecer, com base mais sólida, a correta circunscrição dos gêneros e gru- pos superiores, motivo pelo qual novas pesquisas se mostram necessárias. No presente estudo, a anatomia da madeira de 16 gêneros e 71 espécies da tribo Myrteae foi analisada com o auxílio da Análise de Agrupamento, visando ao reconhecimento de padrões úteis ao esclarecimento de eventuais relações internas no grupo.

\section{MATERIAL E MÉTODOS}

Foram investigadas 71 espécies de 16 gêneros de Myrteae nativos no Brasil (Tabela 1). Os dados qualitativos e quantitativos da estrutura anatômica foram recolhidos de descrições já publicadas por um ou mais dos autores do presente artigo, bem como de outras referências da literatura, as quais se encontram listadas ao final do trabalho (Barros e Callado, 1997; Barros et al., 2001, 2003; Costa, 2012; Paula et al., 2000; Denardi e Marchiori, 2005; Marques et al., 2007; Andrade et al., 2010; Ovando et al., 2010; Santos e Marchiori, 2011b; Santos, 2012; Santos et al., 2013).

Para comparar e reunir tais espécies em grupos de afinidade, realizou-se uma análise de agrupamento hierárquica aglomerativa, com base em caracteres anatômicos de valor taxonômico para Myrtaceae (Tabela 1). Tal análise foi gerada a partir de uma matriz de distância de dissimilaridade de Jaccard, utilizando-se para a geração dos grupos o método de peso proporcional (Unweigthed Pair-Group Method using Arithmetical Averages-UPGMA).

Os dados foram processados no software R, pacote VEGAN (Oksanen et al., 2012). Para a validação do dendrograma resultante, calculouse o coeficiente de correlação cofenético $(r$ de Person). A interpretação e discussão dos resultados levou em consideração a distribuição das espécies e gêneros no dendrograma, em relação ao arranjo tradicional e as mais recentes classificações internas do grupo (Quadro 1).

As características anatômicas utilizadas na análise correspondem, basicamente, às constantes na lista do IAWA Committee (Wheeler et al., 1989). Em certos casos, como largura e altura de raios, no entanto, usou-se classificação 
QUADRO 1 - O arranjo interno de Myrteae, segundo os principais taxonomistas.

\begin{tabular}{|c|c|c|c|c|c|c|c|}
\hline Subtribo & \multicolumn{2}{|c|}{ Myrciinae O. Berg } & \multicolumn{3}{|c|}{ Eugeniinae O. Berg } & \multicolumn{2}{|c|}{ Myrtiinae O. Berb } \\
\hline 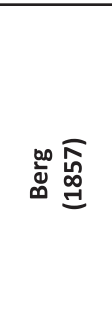 & \multicolumn{2}{|c|}{$\begin{array}{l}\text { "Subtribo Myrcioideae" } \\
\text { Calyptranthes, Gomidesia, } \\
\text { Marlierea, Myrceugenia, } \\
\text { Myrcia, Aulomyrcia } \\
\text { (Myrcia), Calyptromyrcia } \\
\text { (Myrcia), Cerquierea } \\
\text { (Gomidesia), Eugeniopsis } \\
\text { (Marlierea) e Rubachia } \\
\text { (Marlierea) }\end{array}$} & \multicolumn{3}{|c|}{$\begin{array}{c}\text { "Subtribo Eugenioideae" } \\
\text { Calycorectes, Caryophyllus, Eugenia, } \\
\text { Hexachlamys, Mytranthes, } \\
\text { Myrcianthes, Myrciaria, Schizocalyx } \\
\text { (Calycorectes), Siphoneugena, } \\
\text { Syzygium, Aulocarpus (Mouriri - } \\
\text { Melastomataceae), Jambosa } \\
\text { (Syzygium), Phyllocalix (Eugenia) e } \\
\text { Stenocalyx (Eugenia) }\end{array}$} & \multicolumn{2}{|c|}{$\begin{array}{c}\text { "Subtribo Pimentoideae" } \\
\text { Blepharocalyx, Calycolpus, Myrtus e } \\
\text { Psidium. } \\
\text { Abbevillea, Acrandra, Britoa e Lacerdaea } \\
\text { (Campomanesia), Campomanesia, } \\
\text { Pseudocaryophyllus (Pimenta) }\end{array}$} \\
\hline \multirow[t]{2}{*}{ 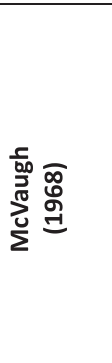 } & \multirow{2}{*}{\multicolumn{2}{|c|}{$\begin{array}{l}\text { "gêneros Mircióides" } \\
\text { Calyptranthes, Gomidesia, } \\
\text { Marlierea, Myrceugenia, } \\
\text { Myrcia, Nothomyrcia } \\
\text { (Myrceugenia) }\end{array}$}} & \multicolumn{3}{|c|}{$\begin{array}{l}\text { "gêneros Eugenióides" } \\
\text { Calycorectes, Eugenia, Hexachlamys, } \\
\text { Myrciaria, Plinia, Siphoneugena }\end{array}$} & \multicolumn{2}{|c|}{$\begin{array}{c}\text { "Psidium e gêneros afins" } \\
\text { Calycolpus, Myrtus, Myrteola, Psidium, } \\
\text { Ugni, Amomyrtus (Myrtus) e Amomyrtella } \\
\text { (Myrtus) }\end{array}$} \\
\hline & & & \multicolumn{3}{|c|}{$\begin{array}{l}\text { "Myrcianthes e gêneros afins" } \\
\text { Legrandia, Luma, Myrcianthes, } \\
\text { Pseudamomis e Reichea }\end{array}$} & \multicolumn{2}{|c|}{ Pseudocaryophyllus e Pimenta } \\
\hline 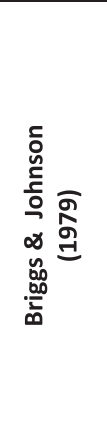 & \multicolumn{2}{|c|}{$\begin{array}{c}\text { “aliança Myrcia” } \\
\text { Calyptranthes, Gomidesia, } \\
\text { Marlierea, Mytranthes, } \\
\text { Myrceugenia, Myrciae } \\
\text { Nothomyrcia } \\
\text { (Myrceugenia) }\end{array}$} & \multicolumn{3}{|c|}{$\begin{array}{c}\text { "aliança Eugenia" } \\
\text { Acreugenia, Calycorectes, Eugenia, } \\
\text { Hexachlamys, Luma (Myrceugenella), } \\
\text { Myrcianthes, Myrciaria, Paramyrciaria, } \\
\text { Plinia, "Pliniopsis", Reichea } \\
\text { (Aspidogenia) e Siphoneugena }\end{array}$} & \multicolumn{2}{|c|}{$\begin{array}{c}\text { "aliança Myrtus" } \\
\text { Acca, Amomyrtella, Amomyrtus, Calycolpus, } \\
\text { Feijoa (Acca), Mosiera, Myrrhinium, } \\
\text { Myrteola, Myrtus, Pimenta, } \\
\text { Pseudocaryophyllus (Pimenta), Psidiopsis } \\
\text { (Calycolpus), Psidium e Ugni }\end{array}$} \\
\hline 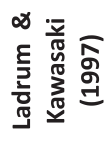 & $\begin{array}{l}\text { Calyptranthes, } N \\
\text { (incluindo Marlie } \\
\text { Gomidesia) } \\
\text { Myrceugeni }\end{array}$ & & $\begin{array}{r}\text { Eu } \\
\text { Hexa } \\
\text { Neon }\end{array}$ & $\begin{array}{l}\text { enia (incluindo C } \\
\text { lamys), Myrcian } \\
\text { incluindo Param } \\
\text { tranthes, Plinia }\end{array}$ & $\begin{array}{l}\text { orectes e } \\
\text { aria), } \\
\text { honeugena }\end{array}$ & $\begin{array}{r}\text { Acca, Accara, Blepharc } \\
\text { Campomanesia, Mos } \\
\text { Pimenta, Psidi }\end{array}$ & $\begin{array}{l}\text { lyx, Calycolpus, } \\
\text { Myrrhinium, } \\
\text { e Ugni }\end{array}$ \\
\hline 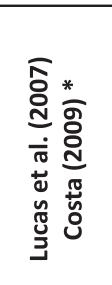 & $\begin{array}{c}\text { "grupo } \\
\text { Pimenta" } \\
\text { Acca, Mosiera } \\
\text { Myrrhinium, } \\
\text { Psidium, Pimenta, } \\
\text { Campomanesia, } \\
\text { Amomyrtus, } \\
\text { Legrandia }\end{array}$ & $\begin{array}{r}\text { "gr } \\
\text { Eug } \\
\text { Eug } \\
\text { Myrc }\end{array}$ & $\begin{array}{l}\text { Ipo } \\
\text { nia" } \\
\text { nia, } \\
\text { inthes }\end{array}$ & $\begin{array}{l}\text { "grupo } \\
\text { Myrteola" } \\
\text { Lenwebbia, } \\
\text { Lophomyrtus, } \\
\text { Neomyrtus, } \\
\text { Ugni, } \\
\text { Myrteola }\end{array}$ & $\begin{array}{r}\text { "grupo } \\
\text { Plinia" } \\
\text { Myrciari } \\
\text { Neomitran } \\
\text { Siphoneug } \\
\text { Plinia }\end{array}$ & $\begin{array}{l}\text { "grupo Myrcia" } \\
\text { Calyptranthes, } \\
\text { Marlierea, } \\
\text { Gomidesia, } \\
\text { Myrcia }\end{array}$ & $\begin{array}{l}\text { "grupo } \\
\text { Myrceugenia" } \\
\text { Blepharocalyx, } \\
\text { Luma, } \\
\text { Myrceugenia }\end{array}$ \\
\hline
\end{tabular}

(Adaptado de: COSTA, 2004)

Os nomes entre parênteses são as atuais sinonímias.

*Não inclui o "grupo Australasiano" reconhecido pelos autores. 
distinta, com vistas a um maior refinamento taxonômico (Tabela 1). Além disso, para atribuir maior confiabilidade aos resultados, optouse por excluir da análise os caracteres anatômicos mais suscetíveis à influência do ambiente, tais como: porosidade; comprimento, diâmetro e frequência de poros; e comprimento e espessura da parede de fibras. Mais informações sobre as espécies em estudo podem ser obtidas nas publicações originais.

\section{RESULTADOS E DISCUSSÃO}

O dendrograma da Figura 1 fornece uma panorâmica da relação entre as espécies em estudo. O nível de distância com que estas se afastam ou se aproximam, reflexo do maior ou menor número de caracteres anatômicos compartilhados, pode ser lido diretamente na vertical, em dissimilaridade, sendo que quanto maior o índice, menor é a proximidade entre as espécies. $\mathrm{O}$ valor do coeficiente de correlação cofenético, de 0,86 , indica que o dendrograma é relevante na análise, de acordo com Valentin (2000).

Como se pode observar no dendrograma, todas as espécies estudadas agruparam-se até por volta de 0,5 de dissimilaridade, sendo que, destas, cerca de $80 \%$ foram agrupadas a menos de 0,3 de dissimilaridade, valores considerados baixos, posto que a distância de Jaccard varia de 0 a 1 (Figura 1). Esse resultado pode ser interpretado como indicativo de grande semelhança estrutural entre as espécies, o que, em última análise, se deve a um elevado número de caracteres anatômicos compartilhados, inclusive entre representantes de gêneros distintos. Entre outros, é o caso de: poros exclusivamente solitários; placas de perfuração simples; pontoações intervasculares alternas; pontoações raio-vasculares semelhantes às intervasculares; parênquima axial seriado; raios heterocelulares, estreitos (até 4 células) e muito numerosos ( $>12 /$ $\mathrm{mm}$ ); e fibrotraqueídeos não septados.

Apesar de não incluídos na análise, são também amplamente recorrentes nas madeiras investigadas, bem como na tribo Myrteae e família Myrtaceae, os seguintes caracteres anatômicos: anéis de crescimento distintos; porosidade difusa; elementos vasculares de comprimento médio; pontoações intervasculares ornamentadas; fibras de comprimento médio e paredes espessas; e ausência de estratificação. Cabe ressaltar que esse conjunto de caracteres nem sempre ocorre simultaneamente, na íntegra, em todas as madeiras, havendo, sempre, todavia, um grande número de caracteres anatômicos em comum, independentemente das espécies em exame.

A grande homogeneidade estrutural, confirmada pela análise, não constitui novidade para a família. Inúmeros trabalhos têm demonstrado a falta de diferenças consistentes entre os distintos taxa, especialmente na tribo Myrteae ou antiga subfamília Myrtoideae (Metcalfe e Chalk, 1972; Dias-Leme et al., 1995; Détienne e Jacquet, 1993; Santos e Marchiori, 2011a; Santos et al., 2013), e não apenas na Anatomia da Madeira (Barroso, 1991; Landrum e Kawasaki, 1997; Sobral, 2003). Como salientado por Santos et al. (2013), esta homogeneidade é mais um indício da estreita relação entre os integrantes da referida tribo.

Dos 16 gêneros investigados, apenas Myrceugenia formou um grupo a parte, com a reunião das quatro espécies estudadas em um mesmo ramo, e, mesmo assim, com um valor relativamente baixo de dissimilidade (Figura 1, seta). Nos demais gêneros, as espécies não se mantiveram unidas e/ou não formaram grupos isolados, fato que se explica tanto pela presença constante de um grande número de caracteres anatômicos compartilhados, quanto pela falta de características genéricas exclusivas. Como exemplo, citam-se os casos de Gomidesia e Myrcia, cujas espécies podem ser tão ou mais dissimilares entre si do que comparado a espécies de gêneros distintos (Figura 1). Este resultado corrobora as observações de Dias Leme et al. (1995), Santos e Marchiori (2011a) e Santos et al. (2013), que também não conseguiram separar os gêneros de Myrteae com base na Anatomia da Madeira, tamanha a semelhança estrutural entre os mesmos. 
TABELA 1 - Distribuição das características anatômicas nas espécies em estudo.

\begin{tabular}{|c|c|c|c|c|c|c|c|c|c|c|c|c|c|c|c|c|c|c|c|c|c|c|c|c|c|}
\hline & \multicolumn{25}{|c|}{ CARACTERES ANATÔMICOS } \\
\hline ESPÉCIES & 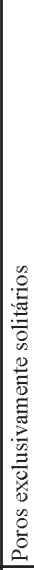 & 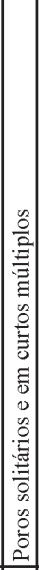 & 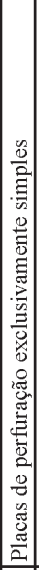 & 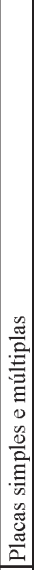 & 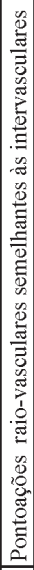 & 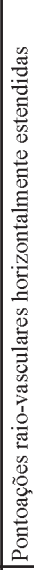 & 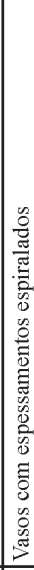 & 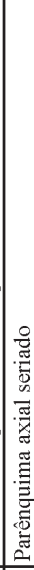 & 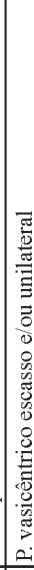 & 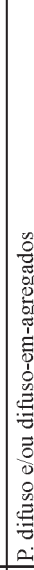 & $\Xi$ & 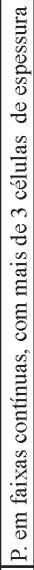 & 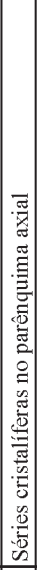 & 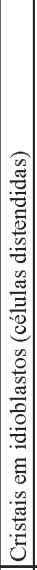 & 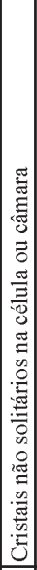 & 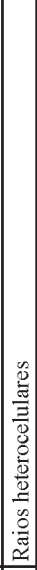 & 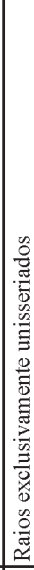 & 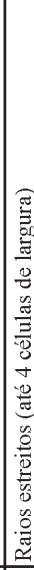 & 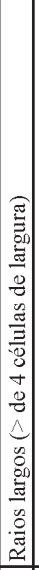 & 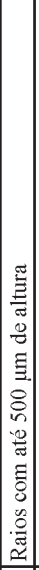 & 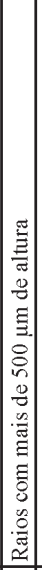 & 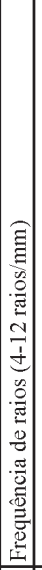 & 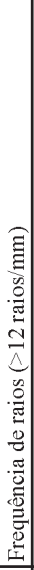 & & 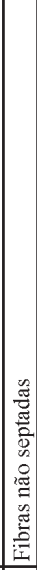 \\
\hline Acca sellowiana ${ }^{9}$ & 1 & 0 & 1 & 0 & 1 & 0 & 0 & 1 & 0 & 1 & 0 & 0 & 1 & 1 & 0 & 1 & 0 & 1 & 0 & 1 & 0 & 0 & 1 & 1 & 1 \\
\hline Blepharocalyx salicifolius ${ }^{9}$ & 1 & 0 & 1 & 0 & 1 & 0 & 0 & 1 & 0 & 1 & 0 & 0 & 1 & 0 & 0 & 1 & 0 & 1 & \begin{tabular}{|l|l|}
0 \\
\end{tabular} & 1 & 0 & 0 & 1 & 1 & 1 \\
\hline \begin{tabular}{|l|} 
Calyptranthes concinna \\
\end{tabular} & 1 & 0 & 1 & 0 & 1 & 0 & 0 & 1 & 0 & 0 & 1 & 0 & 0 & 0 & 0 & 1 & 0 & 1 & 0 & 1 & 0 & 0 & 1 & 1 & 1 \\
\hline \begin{tabular}{|l|l} 
Calyptranthes langsdorffii & ${ }^{2}$ \\
\end{tabular} & 1 & 0 & 1 & 0 & 1 & 0 & 0 & 1 & 0 & 0 & 1 & 0 & 1 & 0 & 0 & 1 & 0 & 1 & 0 & 1 & 0 & 0 & 1 & 1 & 1 \\
\hline \begin{tabular}{|l|} 
Calyptranthes tricona \\
${ }^{9}$ \\
\end{tabular} & 1 & 0 & 1 & 0 & 1 & 0 & 0 & 1 & 0 & 0 & 1 & 0 & 0 & 0 & 0 & 1 & 0 & 1 & \begin{tabular}{|l|l|}
0 \\
\end{tabular} & 1 & 0 & 0 & 1 & 1 & 1 \\
\hline 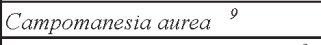 & 1 & 0 & 1 & 0 & 1 & 0 & 0 & 1 & 0 & 1 & 0 & 0 & 0 & 0 & 0 & 1 & 0 & 1 & \begin{tabular}{|l|l|}
0 \\
\end{tabular} & 1 & 0 & 0 & 1 & 1 & 1 \\
\hline \begin{tabular}{|l} 
Campomanesia guazumifolia \\
\end{tabular} & 1 & 0 & 1 & 0 & 1 & 0 & 0 & 1 & 0 & 1 & 0 & 0 & 0 & 0 & 0 & 1 & 0 & 1 & \begin{tabular}{|l|l|}
0 \\
\end{tabular} & 1 & 0 & 0 & 1 & 1 & 1 \\
\hline \begin{tabular}{|c|} 
Campomanesia rhombea \\
\end{tabular} & 1 & 0 & 1 & 0 & 1 & 0 & 0 & 1 & 0 & 1 & 0 & 0 & 0 & 0 & 0 & 1 & 0 & 1 & \begin{tabular}{|l|l|}
0 \\
\end{tabular} & 1 & 0 & 0 & 1 & 1 & 1 \\
\hline Campomanesia xanthocarpa ${ }^{9}$ & 1 & 0 & 1 & 0 & 1 & 0 & 0 & 1 & 0 & 1 & 0 & 0 & 0 & 0 & 0 & 1 & 0 & 1 & 0 & 1 & 0 & 0 & 1 & 1 & 1 \\
\hline Eugenia burkartiana ${ }^{9}$ & 1 & 0 & 1 & 0 & 1 & 0 & 0 & 1 & 0 & 1 & 0 & 0 & 1 & 1 & 0 & 1 & 0 & 1 & 0 & 1 & 0 & 0 & 1 & 1 & 1 \\
\hline Eugenia cachoeirensis ${ }^{3}$ & 1 & 0 & 1 & 0 & 1 & 0 & 0 & 1 & 0 & 0 & 1 & 0 & 1 & 0 & 0 & 1 & 0 & 1 & 0 & 11 & 0 & 0 & 1 & 1 & 1 \\
\hline Eugenia chlorophylla ${ }^{3}$ & 1 & 0 & 1 & 0 & 1 & 0 & 0 & 1 & 0 & 0 & 1 & 0 & 1 & 0 & 0 & 1 & 0 & 1 & 0 & 1 & 0 & 1 & 0 & 1 & 1 \\
\hline Eugenia cuprea $^{2}$ & 1 & 0 & 1 & 0 & 1 & 0 & 0 & 1 & 0 & 1 & 0 & 0 & 1 & 0 & \begin{tabular}{|l|l|}
0 \\
\end{tabular} & 1 & 0 & 1 & 0 & 1 & 0 & 1 & 0 & 1 & 1 \\
\hline Eugenia copacabanensis ${ }^{6}$ & 0 & 1 & 1 & 0 & 1 & 0 & 0 & 1 & 0 & 0 & 0 & 1 . & 1 & 0 & 0 & 1 & 0 & 1 & 0 & 1 & 0 & 0 & 1 & 1 & 1 \\
\hline Eugenia hiemalis ${ }^{9}$ & 1 & 0 & 1 & 0 & 1 & 0 & 0 & 1 & 0 & 0 & 1 & 0 & 1 & 1 & 0 & 1 & 0 & 1 & 0 & 1 & 0 & \begin{tabular}{|l|l|}
0 \\
\end{tabular} & 1 & 1 & 1 \\
\hline Eugenia involucrata ${ }^{9}$ & 1 & 0 & 1 & 0 & 1 & 0 & 0 & 1 & 0 & 1 & 0 & 0 & 1 & 0 & 0 & 1 & 0 & 1 & 0 & 1 & 0 & 0 & 1 & 1 & 1 \\
\hline Eugenia macahensis ${ }^{2}$ & 1 & 0 & 1 & 0 & 1 & 0 & 0 & 1 & 0 & 1 & 0 & 0 & 1 & 0 & \begin{tabular}{|l|l}
0 \\
\end{tabular} & 1 & 0 & 1 & 0 & 0 & 1 & \begin{tabular}{|l|}
0 \\
\end{tabular} & 1 & 1 & 1 \\
\hline Eugenia mansoi ${ }^{9}$ & 1 & 0 & 1 & 0 & 1 & 0 & 0 & 1 & 0 & 0 & 1 & 0 & 1 & 1 & 0 & 1 & 0 & 1 & 0 & 1 & 0 & \begin{tabular}{|l|l|}
0 \\
\end{tabular} & 1 & 1 & 1 \\
\hline Eugenia rostrifolia ${ }^{9}$ & 0 & 1 & 1 & 0 & 1 & 0 & 0 & 1 & 0 & 0 & 0 & 1 & 1 & 1 & \begin{tabular}{|l|}
0 \\
\end{tabular} & 1 & 0 & 1 & 0 & 1 & 0 & 0 & 1 & 1 & 1 \\
\hline Eugenia schuechiana & 1 & 0 & 1 & 0 & 1 . & 0 & 0 & 1 & 0 & 0 & 1 & 0 & 1 & 1 & 0 & 1 & 0 & 1 & 0 & 1 & 0 & 0 & 1 & 1 & 1 \\
\hline Eugenia sonderiana ${ }^{4}$ & 0 & 1 & 1 & 0 & 1 & 0 & 0 & 1 & 0 & 0 & 0 & 1 & 1 & 1 & 0 & 1 & 0 & 1 & 0 & 1 & 0 & \begin{tabular}{|l|}
0 \\
\end{tabular} & 1 & 1 & 1 \\
\hline Eugenia uniflora ${ }^{9}$ & 1 & 0 & 1 & 0 & 1 & 0 & 0 & 1 & 0 & 0 & 0 & 1 & 1 & 1 & 0 & 1 & 0 & 1 & 0 & 1 & 0 & 0 & 1 & 1 & 1 \\
\hline Eugenia uruguayensis ${ }^{9}$ & 1 & 0 & 1 & 0 & 1 & 0 & 0 & 1 & 0 & 1 & 0 & 0 & 1 & 1 & \begin{tabular}{|l|}
0 \\
\end{tabular} & 1 & 0 & 1 & 0 & 1 & 0 & 0 & 1 & 1 & 1 \\
\hline Gomidesia affinis ${ }^{{ }^{3}}$ & 1 & 0 & 1 & 0 & 1 & 0 & 0 & 1 & 0 & 1 & 0 & 0 & 0 & 0 & 0 & 1 & 0 & 0 & 1 & 1 & 1 & 1 & 0 & 1 & 1 \\
\hline Gomidesia anacardifolia ${ }^{?}$ & 1 & 0 & 1 & 0 & 1 & 0 & 0 & 1 & 0 & 1 & 0 & 0 & 0 & 0 & 0 & 1 & 0 & 1 & 0 & 1 & 0 & 1 & 0 & 1 & 1 \\
\hline Gomidesia flagellaris ${ }^{7}$ & 0 & 1 & 0 & 1 & 1 & 0 & 0 & 1 & 0 & 1 & 0 & 0 & 0 & 0 & 0 & 1 & 0 & 1 & 0 & 1 & 0 & 1 & 0 & 1 & 1 \\
\hline Gomidesia palustris $^{9}$ & 1 & 0 & 1 & 0 & 1 & 0 & 0 & 1 & 0 & 1 & 0 & 0 & 0 & 0 & 0 & 1 & 0 & 0 & 1 & 0 & 1 & 0 & 1 & 1 & 1 \\
\hline Gomidesia spectabilis & 1 & 0 & 0 & 1 & 1 & 0 & 0 & 1 & 0 & 1 & 0 & 0 & 0 & 0 & 0 & 1 & 0 & 1 & 0 & \begin{tabular}{|l|} 
\\
\end{tabular} & 0 & 1 & 0 & 1 & 1 \\
\hline Hexachlamys edulis ${ }^{9}$ & 1 & 0 & 1 & 0 & 1 & 0 & 0 & 1 & 0 & 0 & 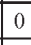 & 1 & 1 & 0 & 0 & 1 & 0 & 1 & 0 & 1 & 0 & 0 & 1 & 1 & 1 \\
\hline Myrcengenia euosma ${ }^{9}$ & 1 & 0 & 0 & 1 & 0 & 1 & 1 & 1 & 0 & 1 & 0 & 0 & 0 & 0 & 0 & 1 & 0 & 1 & 0 & 0 & 1 & 0 & 1 & 1 & 1 \\
\hline Myrcengenia glaucescens ${ }^{9}$ & 1 & 0 & 0 & 1 & 0 & 1 & 1 & 1 & 0 & 1 & 0 & 0 & 0 & 0 & 0 & 1 & 0 & 1 & 0 & 1 & 0 & 0 & 1 & 1 & 1 \\
\hline Myrcengenia miersiana ${ }^{9}$ & 1 & 0 & 0 & 1 & 0 & 1 & 1 & 1 & 0 & 1 & 0 & 0 & 0 & 0 & 0 & 1 & 0 & 1 & 0 & 0 & 1 & 0 & 1 & 1 & 1 \\
\hline Myrcengenia myrtoides ${ }^{9}$ & 1 & 0 & 1 & 0 & 0 & 1 & 1 & 1 & 0 & 1 & 0 & 0 & 0 & 0 & 0 & 1 & 0 & 1 & 0 & 1 & 0 & 0 & 1 & 1 & 1 \\
\hline Myrcia albotomentosa ${ }^{8}$ & 1 & & 1 & 0 & 1 & 0 & 0 & & 0 & & & 0 & 0 & 0 & \begin{tabular}{|l|l} 
\\
\end{tabular} & 1 & & 0 & & & & & & & \\
\hline
\end{tabular}




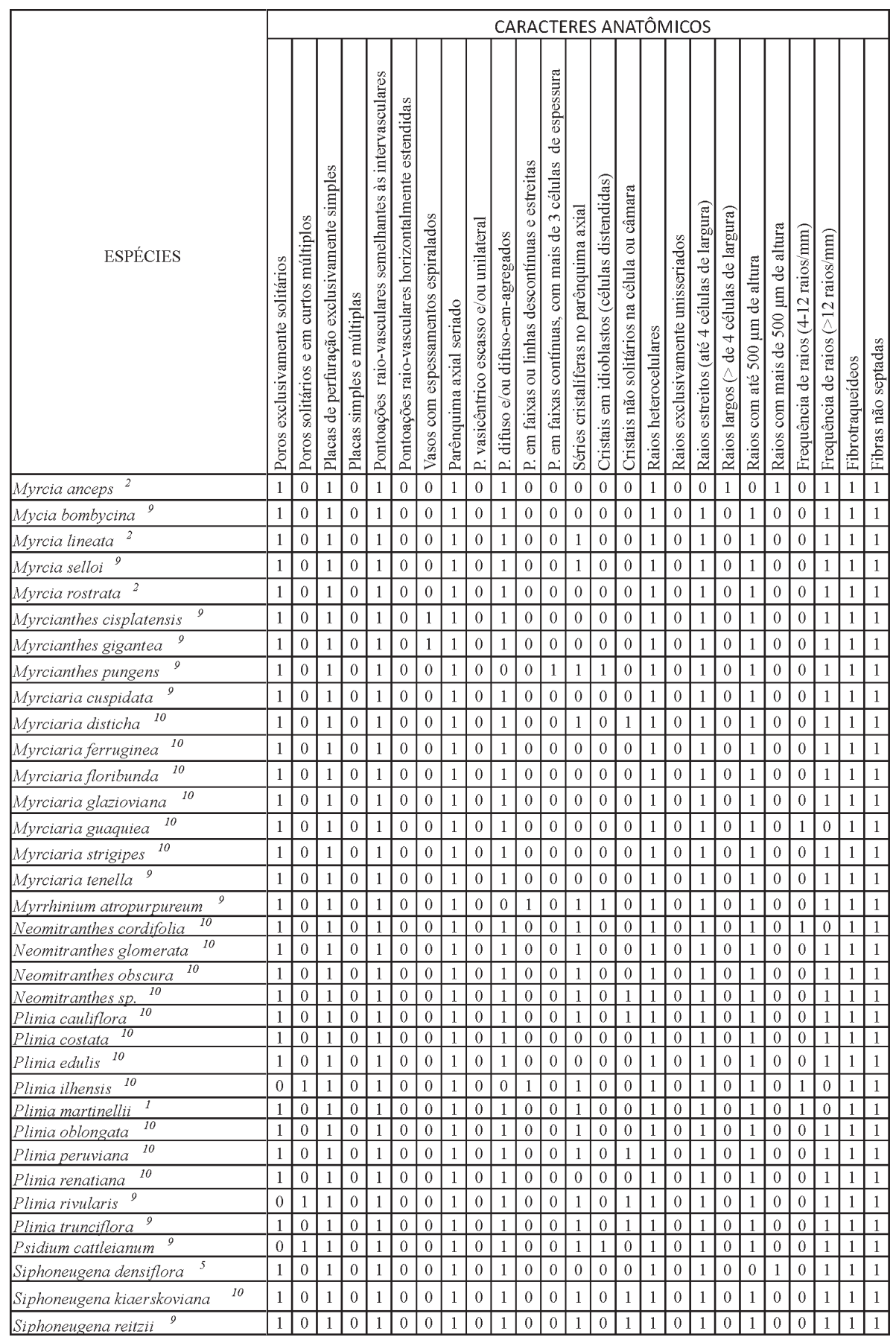

LEGENDA:

Caráter presente $=1$; caráter ausente $=0$.

O número sobrescrito ao epíteto específico designa a referência que originou os dados, a saber:

1 - Barros e Callado (1997); 2 - Barros et al. (2001); 3 - Barros et al. (2003); 4 - Costa (2012); 5 - Paula et al. (2000); 6 - Marques et al. (2007); 7 - Andrade et al. (2010); 8 - Ovando et al. (2010); 9 - Santos e Marchiori (2011b); 10 - Santos et al. (2013). 


\section{Cluster Dendrogram}

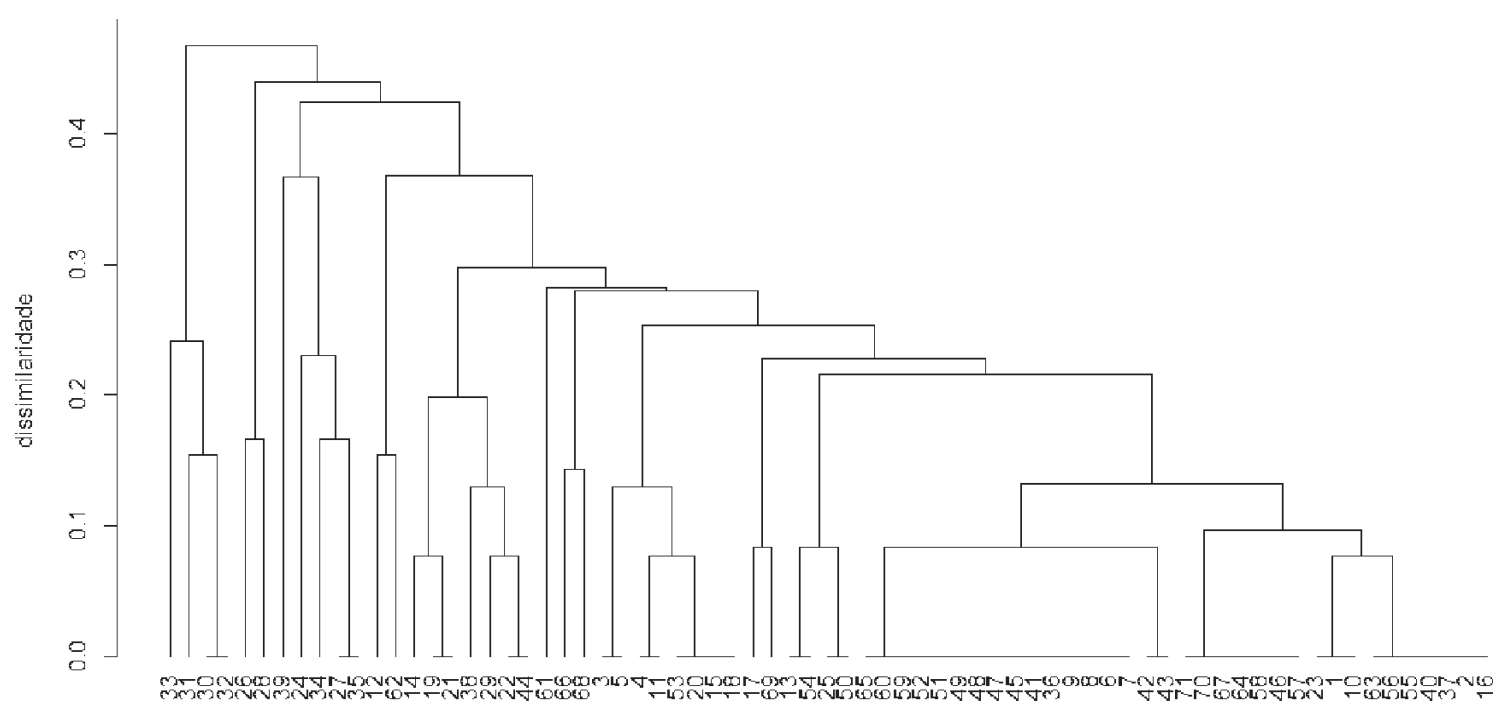

Jaccard

holust (", "average")

FIGURA 1 - Dendrograma das 71 Myrtoídeas investigadas. 33-Myrceugenia myrtoides O.Berg, 31-Myrceugenia glaucescens (Cambess.) D. Legrand \& Kausel, 30-Myrceugenia euosma (O. Berg) D. Legrand, 32-Myrceugenia miersiana (Gardner) D. Legrand \& Kausel, 26-Gomidesia flagellaris D. Legrand, 28-Gomidesia spectabilis O. Berg, 39-Myrcia pubipetala Miq., 24-Gomidesia affinis (Cambess.) D. Legrand, 34-Myrcia albotomentosa DC., 27-Gomidesia palustris (DC.) D. Legrand, 35-Myrcia anceps O. Berg, 12-Eugenia chlorophylla O. Berg, 62-Plinia martinellii G. M. Barroso \& M. Peron, 14-Eugenia copacabanensis Kiaersk., 19-Eugenia rostrifolia D. Legrand, 21-Eugenia sonderiana O. Berg, 38-Myrcia multiflora DC., 29-Hexachlamys edulis (O. Berg) Kausel \& D.Legrand, 22-Eugenia uniflora L., 44-Myrcianthes pungens (O. Berg) D. Legrand, 61-Plinia ilhensis G. M. Barroso, 66-Plinia rivularis (Cambess.) Rotman, 68-Psidium cattleianum Sabine, 3-Calyptranthes concinna DC., 5-Calyptranthes tricona D. Legrand, 4-Calyptranthes langsdorffii O. Berg, 11-Eugenia cachoeirensis O. Berg, 53-Myrrhinium atropurpureum Schott, 20-Eugenia schuechiana O. Berg, 15-Eugenia hiemalis Cambess, 18-Eugenia mansoi O. Berg, 17-Eugenia macahensis Glaz., 69-Siphoneugena densiflora O. Berg, 13-Eugenia cuprea (O. Berg) Mattos., 54-Neomitranthes cordifolia (D. Legrand) D. Legrand, 25-Gomidesia anacardifolia (Gardner) O. Berg, 50-Myrciaria guaquiea (Kiaersk.) Mattos \& D. Legrand, 65-Plinia renatiana G. M. Barroso \& Peixoto, 60-Plinia edulis (Vell.) Sobral, 59-Plinia costata Amshoff, 52-Myrciaria tenella O. Berg, 51-Myrciaria strigipes O. Berg, 49-Myrciaria glazioviana (Kiaersk.) G. M. Barroso ex Sobral, 48-Myrciaria floribunda O. Berg, 47Myrciaria ferruginea $\mathrm{O}$. Berg, 45-Myrciaria cuspidata O. Berg, 41-Myrcia rostrata DC., 36-Myrcia bombycina Kiaersk., 9-Campomanesia xanthocarpa O. Berg, 8-Campomanesia rhombea O. Berg, 6-Campomanesia aurea O. Berg, 7Campomanesia guazumifolia Blume, 42-Myrcianthes cisplatensis (Cambess.) O. Berg, 43-Myrcianthes gigantea (D. Legrand) D. Legrand, 71-Siphoneugena reitzii D. Legrand, 70-Siphoneugena kiaerskoviana (Burret) Kausel, 67-Plinia trunciflora (O. Berg) Kausel, 64-Plinia peruviana (Poir.) Govaerts, 58-Plinia cauliflora (Mart.) Kausel, 46-Myrciaria disticha O. Berg, 57-Neomitranthes sp., 23-Eugenia uruguayensis Cambess., 1-Acca sellowiana (O. Berg) Burret, 10Eugenia burkartiana (D. Legrand) D. Legrand, 63-Plinia oblongata (Mattos) Mattos, 56-Neomitranthes obscura (DC.) N. Silveira, 55-Neomitranthes glomerata (D. Legrand) D. Legrand, 40-Myrcia selloi (Spreng.) N. Silveira, 37-Myrcia lineata Nied., 2-Blepharocalyx salicifolius (Kunth) O. Berg, 16-Eugenia involucrata DC. $r=0,86$ 
A segregação de Myrceugenia dos demais gêneros e espécies em estudo deve-se à presença de um conjunto de caracteres de distribuição mais restrita na família, salientando-se: vasos com espessamentos espiralados; placas de perfuração múltiplas; e pontoações raio-vasculares horizontalmente estendidas. Tal singularidade já era esperada, em virtude da existência de uma série de estudos anteriores que apontavam nesta direção, incluindo Anatomia da Madeira (Record e Hess, 1949; Schmid e Baas, 1984; Santos e Marchiori, 2011a), morfologia (Landrum, 1981) e análise de DNA (Lucas et al., 2005; Costa, 2009). Myrceugenia, aliás, devido à combinação exclusiva dos três caracteres acima, é tido por alguns autores como o único gênero em Myrteae que é passível de identificação anatômica (Schmid e Baas, 1984; Santos e Marchiori, 2011a).

Outra questão envolvendo Myrceugenia diz respeito a sua relação com outros dois gêneros (Blepharocalyx e Luma) do chamado "grupo Myrceugenia" sensu Lucas et al. (2007) (Quadro 1). A análise do dendrograma, bem como da anatomia da madeira dos dois taxa incluídos no presente estudo, não apoia a permanência de Blepharocalyx no referido grupo, em consonância com a interpretação de Murillo et al. (2012), que também não consideram o "grupo Myrceugenia" monofilético.

Acerca da discussão sobre a validade e/ou abrangência de alguns gêneros, tais como Hexachlamys e Eugenia, Myrcia e Gomidesia, Plinia e Myrciaria, Neomitranthes e Siphoneugena, o presente estudo não permite contestar, nem tampouco confirmar as alterações sugeridas, haja vista a distribuição difusa e grande proximidade das espécies, evidenciadas pelo dendrograma. Em outras palavras, falta delimitação genérica para todos os grupos envolvidos, com exceção de Myrceugenia.

Dias-Leme et al. (1995), em estudo da subtribo Myrciinae (fora Myrceugenia), também não conseguiram separar os gêneros, concluindo, por este motivo, que a inclusão de Calyptranthes, Gomidesia, Marlierea e Myrcia em um único gênero (Myrcia), como proposto por McVaugh (1968,), é suportada pela anatomia da madeira. No presente trabalho, de modo semelhante a Santos e Marchiori (2011a) e Santos et al. (2013), prefere-se concluir que, em virtude da grande homogeneidade estrutural, torna-se limitado o uso da anatomia da madeira em discussões desta natureza, pelo menos na tribo Myrteae. Distintamente de Dias-Leme et al. (1995), conclui-se que todos os gêneros incluídos no presente estudo, com exceção de Myrceugenia, poderiam ser reunidos no mesmo grupo taxonômico, o que, de fato, não parece razoável.

Apesar da falta de limites claros para os gêneros, reflexo da ausência de caracteres anatômicos exclusivos, aventou-se a possibilidade de que as espécies, mesmo assim, pudessem agrupar-se em categorias taxonômicas superiores e, portanto, menos restritivas, refletindo classificações supragenéricas existentes dentro da tribo Myrteae ou subfamília Myrtoideae. Esta expectativa, no entanto, também não se confirma. Como no caso dos gêneros, não é possível reconhecer agrupamentos infratribais compatíveis com qualquer classificação do grupo, seja antiga ou atual. É que, devido à grande homogeneidade estrutural das espécies, as diferenças apontadas pela análise morfológica e molecular não repercutem da mesma forma na anatomia da madeira, como demonstrado claramente pela análise de agrupamento das 71 espécies investigadas (Figura 1). Caso se dividisse arbitrariamente, por exemplo, o conjunto das espécies em estudo ao valor de 0,4 de dissimilaridade, ainda assim mais de $80 \%$ dos gêneros ficariam posicionados no mesmo grupo, longe, portanto, de qualquer arranjo atualmente conhecido.

Em suma, os resultados do presente estudo, bem como a análise de referências da literatura para espécies da tribo Myrteae, permitem concluir que não há parâmetros anatômicos consistentes para o reconhecimento de gêneros (exceto Myrceugenia) e grupos superiores, o que limita o uso da anatomia da madeira em questões 
taxonômicas da referida tribo. Tal uniformidade estrutural, por outro lado, depõe favoravelmente à interpretação de estudos filogenéticos recentes, que consideram a Tribo Myrteae como um grupo natural (Wilson et al.,2001; Lucas et al., 2007; Costa, 2009).

\section{REFERÊNCIAS BIBLIOGRÁFICAS}

ANDRADE, I.M.; LONGUI, E.L; SANTINI JUNIOR, L.; SOBRAL, E.S.; AGUIAR, O.T.; FLORSHEIM, S.M.B.; LIMA, I.L. Anatomia comparada do lenho de quatro espécies de Gomidesia spp. Berg. (Myrtaceae) do Parque Estadual Carlos Botelho-SP. $4^{\circ}$ Seminário de Iniciação Científica do Instituto Florestal. 2010.

BARROS, C.F.; CALLADO, C.H. Madeiras da Mata Atlântica. Anatomia do lenho de espécies ocorrentes nos remanescentes florestais do estado do Rio de Janeiro, Brasil. Rio de Janeiro: Instituto de Pesquisas Jardim Botânico do Rio de Janeiro, 1997. $86 \mathrm{p}$.

BARROS, C.F.; CALLADO, C.H.; MARCON, M.L.; COSTA, C.G.; CUNHA, M.; MARQUETE, O. Madeiras da Mata Atlântica. Anatomia do lenho de espécies ocorrentes nos remanescentes florestais do estado do Rio de Janeiro, Brasil. Rio de Janeiro: Instituto de Pesquisas Jardim Botânico do Rio de Janeiro, 2001. 94 p.

BARROS, C.F.; CALLADO, C.H.; CUNHA, M.; MARCON-FERREIRA, M.L.; TAMAIO, N.; MARQUETE, O.; COSTA, C.G. Madeiras da Mata Atlântica. Anatomia do lenho de espécies ocorrentes nos remanescentes florestais do estado do Rio de Janeiro, Brasil. Rio de Janeiro: Instituto de Pesquisas Jardim Botânico do Rio de Janeiro, 2003. 86 p.

BARROSO, G.M. Sistemática de Angiospermas do Brasil. Viçosa: UFV, 1991. 377 p.

BERG, O. Revisio Myrtacearum Americae. Linnaea, v. 27 , p. 1-472. 1855-56.

BERG. O. Myrtaceae. In: MARTIUS, C.F.P. von. Flora Brasiliensis, v. 14, p. 1 - 655. 1857-59.

COSTA, I.R. Estudos cromossômicos em espécies de Myrtaceae Juss. no sudeste do Brasil. 2004. 80 f. Dissertação de Mestrado - Universidade Estadual de Campinas (UNICAMP), Campinas, 2004.
COSTA, I.R. Estudos evolutivos em Myrtaceae: aspectos citotaxonômicos e filogenéticos em Myrteae, enfatizando Psidium e gêneros relacionados. 2009. 235 f. Tese (Doutorado) - Universidade Estadual de Campinas, Campinas, 2009.

COSTA, L.,M.,S. Anatomia ecológica e dendrologia de Eugenia sonderiana e Eucalyptus saligna na Serra do Cipó - Minas Gerais. 2012. 71 f. Dissertação (Mestrado) - Universidade Federal de Minas Gerais, Belo Horizonte, 2012.

DENARDI, L.; MARCHIORI, J.N.C. Anatomia ecológica da madeira de Blepharocalyx salicifolius (H.B.K.) Berg. Ciência Florestal, Santa Maria, v. 15, n. 2, p. 119-127, 2005.

DÉTIENNE, P.; JACQUET, P. Atlas d'identification des bois de l'Amazonie et des regions voisines. Centre Technique Forestier Tropical, Nogentsur-Marne, 1983. $640 \mathrm{p}$.

DIAS-LEME, C.L.; GASSON, P.; LUGHADA, E.N. Wood anatomy of four Myrtaceae genera in the subtribe Myciinae from South America. IAWA Bulletin, n. 16, p. 87-95, 1995.

JOHNSON, L.A.S.; BRIGGS, B.G. Myrtales and Myrtaceae: a phylogenetic analysis. Annals of the Missouri Botanical Garden, v. 71, p. 700756, 1984.

GOVAERTS, R. et al. World Checklist of selected plant families - Myrtaceae. Kew Publishing, Royal Botanic Gardens, Kew. 2008.

LANDRUM, L.R. A monograph of the genus Myrceugenia. Flora Neotropica, n. 29, p. 1-137, 1981.

LANDRUM, L.R.; KAWASAKI, M.L. The genera of Myrtaceae in Brasil: an illustrated synoptic treatment and identification keys. Brittonia, n. 49, p. 508-536, 1997.

LUCAS, E.J.; SOBRAL, M. Proposal to conserve the name Myrcia gainst Calyptranthes (Myrtaceae). Taxon, v. 60, p. 605, 2011.

LUCAS, E.J. et al. Phylogenetic patterns in the fleshyfruited Myrtaceae - preliminary molecular evidence. Plant Systematics and Evolution, v. 251, p. 35-51, 2005.

LUCAS, E.J. et al. Suprageneric phylogenetics of Myrteae, the generically richest tribe in Myrtaceae (Myrtales). Taxon, v. 56, p. 11051128, 2007.

LUCAS, E.J. et al. Phylogenetics, morphology and evolutions of the large genus Myrcia s. $l$. 
(Myrtaceae). International Journal of Plant Sciences, v. 172, p. 915-934, 2011.

LUCAS, E.J. et al. Suprageneric Phylogenetics of Myrteae, the Generically Richest Tribe in Myrtaceae (Myrtales). Taxon, v. 56, p. 11051128, 2007.

MARQUES, P. A.; ARAÚJO, G. U. C.; BARROS, C. F.; CALLADO, C. H. Anatomia do lenho de três espécies de Eugenia L. (Myrtaceae) de mata e restinga. Revista Brasileira de Biociências, Porto Alegre, v. 5, supl. 1, p. 801-803, 2007.

McVAUGH, R. The genera of American Myrtaceae, an interim report. Taxon, v. 17, n. 8, p. 354-418, 1968.

METCALFE, C.R.; CHALK, L. Anatomy of the Dicotyledons. Oxford: Clarendon Press, 1972, $1500 \mathrm{p}$.

MURILO, J.; RUIZ, E.; LANDRUM, L.R.; STUESSY, T.F.; BARFUSS, M.H.J. Phylogenetic relationships in Myrceugenia (Myrtaceae) based on plastid and nuclear DNA sequences. Molecular Phylogenetics and Evolution, v. 62, n. 2, p.764-776, 2012.

OVANDO, P.C. et al. Anatomia do lenho de Myrcia albotomentosa DC. e Myrcia multiflora (Lam.) DC. (Myrtaceae). $4^{\circ}$ Seminário de Iniciação Científica do Instituto Florestal, 2010.

OKSANEN, J.; BLANCHET, F. G.; KINDT, R.; LEGENDRE, P.; O'HARA, R. B.; SIMPSON, G. L.; STEVENS, M. H. H.; WAGNER, H. Vegan: community ecology package. Version 2.0-6. 2012. Available from: <http://vegan.r-forge.r-project.org/ $>$. Acesso em: 22/06/2012.

PAULA, J.E.; JUNIOR, F.G.S.; SILVA, A.P.P. Caracterização anatômica de madeiras nativas de matas ciliares do centro-oeste brasileiro. Scientia Forestalis, v. 58, p. 73-89, 2000.

RECORD, S.J.; HESS R.W. Timbers of the New World. New Haven: Yale University Press, 1949. $640 \mathrm{p}$

SANTOS, S.R. Contribuição ao estudo anatômico das Myrtaceae nativas no Rio Grande do Sul. 2012. 142 p. Tese de Doutorado - Programa de Pós-Graduação em Engenharia Florestal, Uni- versidade Federal de Santa Maria, Santa Maria, RS, Brasil, 2012.

SANTOS, S.R.; MARCHIORI, J.N.C. A classificação interna da tribo Myrteae, sob o ponto de vista da anatomia da madeira. Balduinia, n. 32, p. 1734, 2011a.

SANTOS, S.R.; MARCHIORI, J.N. Madeiras do Rio Grande do Sul 4 - Descrição microscópica de 34 Mirtáceas nativas. Santa Maria: Anaterra, 2011b. 80p.

SANTOS, G.; CALLADO, C.; SOUZA, M.; COSTA, C. Wood anatomy of Myrciaria, Neomitranthes, Plinia e Siphoneugena species (Myrteae, Myrtaceae). IAWA Journal, v.34, n. 3, p. 313-323, 2013.

SCHMID, R.; BAAS, P. The occurrence of scalariform perforation plates and helical thickenings in wood of Myrtaceae. IAWA Bulletin, v. 5, n. 3, p. 197-215, 1984.

SOBRAL, M. A família Myrtaceae no Rio Grande do Sul. São Leopoldo: Unisinos, 2003. 215 p.

SOBRAL, M.; PROENÇA, C.; SOUZA, M.; MAZINE, F.; LUCAS, E. Myrtaceae in Lista de Espécies da Flora do Brasil. Jardim Botânico do Rio de Janeiro. Disponível em: <http:// floradobrasil.jbrj.gov.br/jabot/floradobrasil/ FB171>. Acesso em: 06 Out. 2014.

VALENTIN, J. L. Ecologia numérica: uma introdução à análise multivariada de dados ecológicos. Rio de Janeiro: Interciência, 2000. 117 p.

WHEELER, E.A.; BAAS, P.; GASSON, P.E. IAWA list of microscopic features for hardwood identification. IAWA Bull., v.10, n. 3, p. 218-359, 1989.

WILSON, P.G.; O’BRIEN, M.M.; GADEK, P.A.; QUINN, C.J. Myrtaceae revisited: a reassessment of infrafamilial groups. American Journal of Botany, v. 88, n. 11, p. 2013-2015, 2001.

WILSON, P.G.; O'BRIEN, M.M.; HESLEWOOD, M.M.; QUINN, C.J. Relationship within Myrtaceae sensu lato based on matk phylogeny. Plant Systematics and Evolution, v. 251, p. 319, 2005. 